\title{
Neonatal lupus erythematosus
}

\author{
Wei Liu MD, Dong-Lai Ma MD PhD
}

Cite as: CMAJ 2020 February 18;192:E163. doi: 10.1503/cmaj.191172

A 5-week-old infant boy presented to the dermatology department with a 3-week history of annular plaques. The patient had multiple papules and annular plaques with a raised border on his scalp and face (Figure 1). His mother had an uncomplicated pregnancy, although she had a history of systemic lupus erythematosus. She had no flares during pregnancy and was not on medication.

The patient had normal white blood cell counts and renal function but increased levels of alanine transaminase 67.5 [normal range, $0-40$ ] $\mathrm{U} / \mathrm{L}$ ) and aspartate transaminase (73.6 [normal range, 0-40] U/L). Results for testing for antinuclear antibodies were positive (1:1280 titre in speckled pattern), and strongly positive for anti-SSA/Ro and anti-SSB/La autoantibodies. Results for abdominal ultrasonography (conducted at the mother's request to rule out other adominal diseases) and electrocardiography were normal. His mother tested positive for ANA with a (1:1280 titre in speckled pattern), and strongly positive for anti-SSA/Ro and antiSSB/La autoantibodies.

We diagnosed neonatal lupus erythematosus and advised that sun protection be used but did not suggest other interventions. At 3-month follow-up, the patient's lesions had gradually resolved and levels of liver enzymes had decreased.

Neonatal lupus erythematosus is an uncommon acquired autoimmune disease caused by the passive transfer of autoantibodies, especially anti-Ro and anti-La autoantibodies, across the placenta. Mothers usually have known or undiagnosed autoimmune diseases with circulating autoantibodies. ${ }^{1}$ Clinical manifestations of neonatal lupus erythematosus, commonly affecting the skin and heart, can be present at birth or several weeks after birth. ${ }^{2}$ Erythematous patches or plaques in annular or discoid shape usually affect sun-exposed areas; in some cases, periocular erythema (raccoon-eye appearance) may occur, which is considered a distinctive feature. ${ }^{3}$ Congenital heart block is a severe complication, sometimes causing fetal death or necessitating insertion of a permanent pacemaker.

Differential diagnosis includes congenital syphilis, tinea corporis, sarcoidosis, granuloma annulare, Sweet syndrome and urticaria. Noncardiac manifestations usually resolve with clearance of the maternal autoantibodies. Rheumatic or autoimmune diseases may subsequently develop in childhood, and follow-up is suggested until adolescence. ${ }^{4}$ The risk of neonatal lupus erythematosus in children of future pregnancies is increased (36\%-49\%). ${ }^{5}$

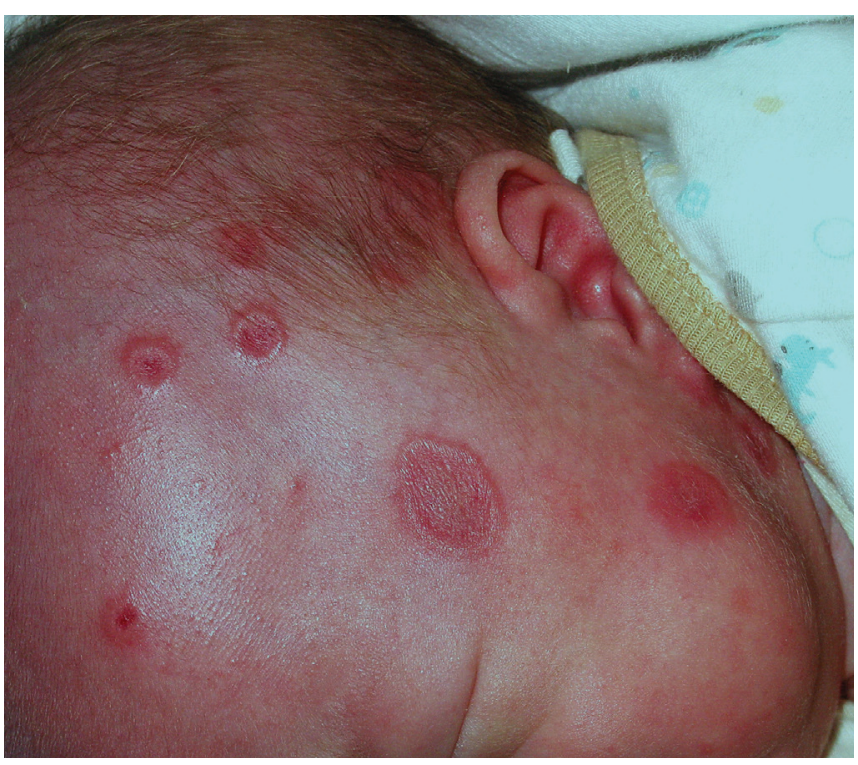

Figure 1: Multiple erythematosus papules and annular plaques with a raised border on the face and scalp of a 5-week-old boy with neonatal lupus erythematosus.

\section{References}

1. Zuppa AA, Riccardi R, Frezza S, et al. Neonatal lupus: follow-up in infants with anti-SSA/Ro antibodies and review of the literature. Autoimmun Rev 2017;16:427-32.

2. Li YQ, Wang Q, Luo Y, et al. Neonatal lupus erythematosus: a review of 123 cases in China. Int J Rheum Dis 2015;18:761-7.

3. Ramphul K, Mejias SG, Ramphul-Sicharam Y. Cutaneous neonatal lupus erythematosus: a case report. Cureus 2018;10:e2212.

4. Martin V, Lee LA, Askanase AD, et al. Long-term followup of children with neonatal lupus and their unaffected siblings. Arthritis Rheum 2002;46:2377-83.

5. Izmirly PM, Llanos C, Lee LA, et al. Cutaneous manifestations of neonatal lupus and risk of subsequent congenital heart block. Arthritis Rheum 2010;62:1153-7.

\section{Competing interests: None declared.}

This article has been peer reviewed.

The authors have obtained parental consent.

Affiliations: Department of Dermatology (Liu, Ma), Peking Union Medical College Hospital, Chinese Academy of Medical Sciences and Peking Union Medical College, Beijing, China

Correspondence to: Dong-Lai Ma, mdonglai@sohu.com 